\title{
Analysis of DMT-FDMA as a Multiple Access Scheme for Broadband Indoor Power-Line Communications
}

\author{
José Antonio Cortés, Luis Díez, Francisco Javier Cañete, José Tomás Entrambasaguas
}

\begin{abstract}
This paper analyzes the performance of a frequency division multiple access (FDMA) scheme for indoor broadband power-line communications based on discrete multitone (DMT) modulation. Bit-rate loss due to multiple access interference (MAI) is assessed for a synchronous and an asynchronous scheme. Improvements obtained by means of pulse-shaping at the transmitter and windowing at the receiver in the presence of symbol and frequency asynchrony are given. Performance is evaluated for carrier assignment strategies based on individual carrier allocation and on the assignation of groups of tones. The analysis is carried out with the help of a novel analytical procedure that allows separate calculation of the distortion terms (intersymbol and intercarrier interference and MAI) of a DMT system operating over a linear time-invariant (LTI) channel. ${ }^{l}$
\end{abstract}

Index Terms - Indoor power-line, DMT-FDMA, multiple access interference, symbol and frequency synchronization.

\section{INTRODUCTION}

Indoor power lines are a feasible and cost-effective solution to provide the broadband data connections required by the high-speed home media applications and the so-called "triple play service" (video, television and internet access) recently offered by DSL (Digital Subscriber Line) operators.

Communication channels established over indoor power lines are frequency and time selective, with remarkably differences between locations even in a specific network. Their frequency response present notches with a priori unknown position and the received noise is strongly coloured [1,2]. Channel characteristics also exhibit a time variation with a twofold origin. The first is caused by the connection and disconnection of electrical devices and represent long-term changes [2]. The second is due to the time-variant behaviour of the electrical devices related to the mains cycle, which produce a cyclic short-time variation in the frequency response and cyclostationary components in the received noise [3].

Discrete Multitone (DMT) modulation is one of the most appropriate transmission schemes to cope with the aforementioned impairments [2,4]. Accordingly, it has been selected by the main alliances involved in the development of

\footnotetext{
1 This work has been supported in part by the Spanish Ministry of Educación y Ciencia under project $\mathrm{n}^{\mathrm{o}}$ TIC2003-06842.

The authors are with the Department of Ingeniería de Comunicaciones, University of Málaga, Málaga 29071 SPAIN (telephone: +34 952137181, e-mail: jaca@ic.uma.es)
}

broadband power-line communications systems $[5,6]$.

Several multiple access strategies can be employed to share the indoor power-line network among different users. Contention schemes, like the CSMA/CA adopted in the IEEE 802.11 wireless LAN standards, do not take into account the channel characteristics of the different users, which may be quite different. Moreover, they are not appropriate to provide the latency, jitter and guaranteed bandwidth needed by some streaming applications. These quality of service (QoS) requirements can be fulfilled by using time-division multiple access (TDMA) or FDMA schemes in which a centralized manager dynamically modifies the time slots or the frequency bands assigned to each user.

In TDMA, the number of time slots of each user is assigned according to its QoS requirements. During these slots, which last several DMT symbols, only one user occupies the available bandwidth. Since unused carriers in one link, because of their low signal-to-noise ratio (SNR), may experience acceptable SNR in other links, this strategy may result in a waste of capacity. To overcome this pitfall, hybrid TDMA-FDMA schemes have been proposed [7].

In FDMA the available bandwidth is shared among the different users. This process results particularly efficient when employing a DMT modulation, since the bands assigned to the different users overlap. Moreover, since in FDMA the protocol data units length is no longer limited by the time slots duration, the transmitted overhead is reduced. On the other hand, it requires symbol and frequency synchronization of all terminals to avoid multiple access interference among users $[8,9]$. However, in environments like Very High Digital Subscriber Line (VDSL), it has been demonstrated that symbol synchronization can be avoided by using pulse-shaping and windowing [9].

TDMA is the multiple access scheme implemented in the current generation of broadband power-line modems, probably due to its simplicity. However, to the authors' knowledge, there is no comprehensive study about the use of a DMTFDMA scheme in an indoor power-line scenario. The aim of this work is to analyze the performance of this strategy in the frequency band up to $30 \mathrm{MHz}$. The effects of the channel dispersion and the symbol and frequency asynchrony on the system performance are assessed. The utilization of a DMT modulation with pulse-shaping and windowing as a mean for reducing the above effects is also studied. The influence of the carrier allocation strategy in the performance of synchronous 
and asynchronous DMT-FDMA systems is evaluated as well.

For simplicity, an LTI channel model is assumed throughout the work. This simplification does not limit the validity of the results in the case of an FDMA system, in which each carrier experiences all the cyclic changes of the channel. When a TDMA system is considered, the LTI assumption is not acceptable, since the bit-rate achieved by each user depends on the position of the assigned time slots within the mains cycle. In addition, only one direction of the communication is considered in each link, i.e. simplex operation is assumed. This is done to avoid the selection of a duplexing scheme, whose choice must take into account the analog-to-digital conversion problem [10].

The study presented in this paper is carried out with the help of a novel analytical method based on the transmission matrix of a DMT system operating over an LTI channel. This procedure, which constitutes one of the contributions of this work, allows separate computation of the different distortion terms (ISI, ICI and MAI).

The paper is organized as follows. Section II summarises the most important aspects of a multiuser DMT scenario: distortion sources, the use of a DMT system with pulseshaping at the transmitter and windowing at the receiver and carrier assignment strategies. In section III the aforementioned analytical method used to evaluate the distortion is presented. In section IV, parameterization of the DMT system with pulseshaping and windowing is assessed. Performance degradation caused by the frequency selectivity of the channel and the symbol and frequency asynchrony is evaluated. Finally, main conclusions drawn for the paper are summarised in section $\mathrm{V}$.

\section{DMT-FDMA MULTIPLE ACCESS SCHEME}

In a centralized DMT-FDMA multiple access scheme, the $N$ carriers of the DMT system are dynamically distributed among the $M$ links by a central entity. Fig. 1 shows a network with two unidirectional active links.

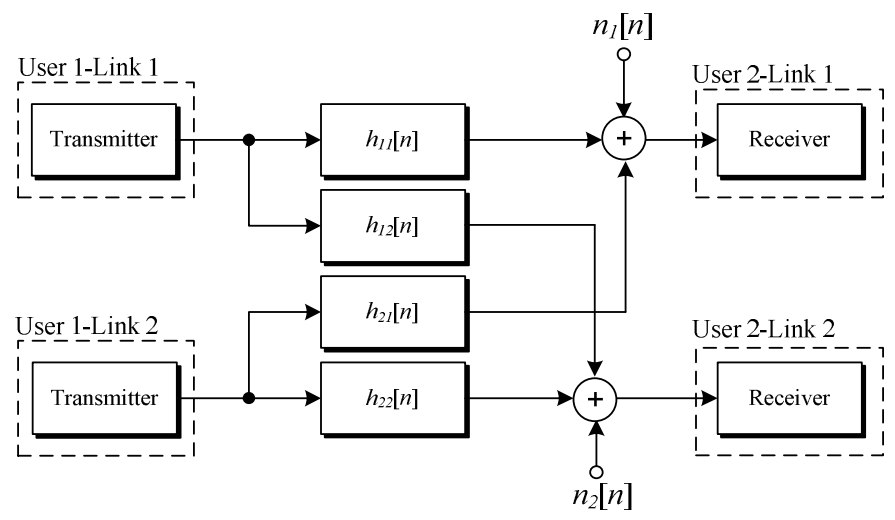

Fig. 1. Simplified discrete-time multiuser environment with two links.

Users and impulse responses are named on a link basis. Thus, $h_{i j}[n]$ denotes the impulse response of the channel seen from the transmitter of user 1-link $i$ to the receiver of user 2link $j$ and $n_{i}[n]$ represents the noise at the receiver of user 2link $i$. From now on, $h_{i j}[n]$ would be referred to as interfered channels and $h_{i i}[n]$ to as desired channels. The reverse directions of the links can be also described in terms of the above impulse responses, since it is a well-known fact that indoor power-line channel responses are symmetric [11].

\section{A. Causes of performance degradation}

MAI is the main cause of performance degradation in a DMT-FDMA system. It may have three different origins: frequency selectivity of the channel, misalignment between the desired and interferer symbols at the input of the desired receiver's DFT and frequency offsets between the clocks of the interferer transmitter and the desired receiver.

MAI caused by the frequency selectivity of the interferer channel can be easily overcome in a synchronized DMT system by using a cyclic prefix length longer than the channel impulse response. However, due to the long impulse responses of indoor power-line channels, a shorter cp must be selected and some residual MAI will exist [2]. This type of distortion constitutes the upper bound for the performance of the DMTFDMA strategy.

In an FDMA scheme with no specific synchronization method, the transmissions of the different users are synchronized only when the central manager performs a new carrier distribution. From that point on, offsets between the clock frequencies of the interferer and interfered users will cause a time-varying symbol asynchrony In an indoor powerline scenario this is the main cause of symbol misalignment, since no significant differences are expected among the propagation delays of links from a specific site due to the small distances involved.

Symbol misalignment and sampling frequency errors cause MAI [8,9] even if the impulse response of the interferer channel is shorter than the $\mathrm{cp}$. In order to quantify their respective effects, this work analyzes separately the performance degradation caused by an integer symbol misalignment $\Delta n$ (samples) additional to the propagation delay difference, and by a $\Delta f$ (ppm) clock frequency mismatch.

In general, the power of the MAI depends on the difference between the attenuation of the interferer and the desired channel, the dispersion of the interferer channel, the magnitude of the symbol misalignment and sampling frequency offset, the shape of pulse used in the transmitter and the distance between the interferer and interfered carrier. Assigning groups of tones instead of individual carriers is a common technique used to reduce MAI [9].

\section{B. DMT with pulse-shaping and windowing}

The discrete-time model of the transmitted signal in a conventional DMT system can be expressed as

$$
x[n]=\sum_{m=-\infty}^{\infty} \sum_{k=-(N-1)}^{N} X_{m, k} e^{j \frac{\pi}{N} k(n-c p-m M)} w_{M}[n-m M],
$$

where $X_{m, k}$ represent the $m$-th complex value transmitted in carrier $k, M=2 N+c p$ and $w_{M}[n]$ is a rectangular window 
with non-zero samples in the interval $0 \leq n \leq M-1$. The complex values transmitted in the $m$-th symbol can be extracted carrying out a $2 N$-point DFT over the sequence $x_{m}[n]=x[n+m M+c p]$ windowed with $w_{2 N}[n]$.

The out of band power captured by the side lobes of $w_{2 N}[n]$ can be reduced by using a non-rectangular window at the receiver. The method proposed in [12] allows to maintain both orthogonality and the DFT structure of the receiver. To this end, the cyclic prefix length is extended with $\beta$ samples more than the ones required to avoid ISI and ICI. The $m$-th received symbol is delayed $\beta / 2$ samples, $x_{m}[n-\beta / 2]$, and windowed with $w_{R X}[n]$, which has non-zero samples only in the interval $-\beta / 2 \leq n \leq 2 N+\beta / 2-1$. As long as

$$
\sum_{r=-\infty}^{\infty} w_{R X}[n-2 N r]=1 \text {, }
$$

the transmitted values, $X_{m, k}$, can be obtained from the samples of the Fourier transform of $x_{m}[n-\beta / 2] w_{R X}[n]$ taken at $\omega_{k}=\pi k / N, k=-(N-1) \ldots N$. These values can be calculated by means of a $2 N$-point DFT over the samples $-\beta / 2 \leq n \leq 2 N+\beta / 2-1$ of the time-aliased signal

$$
\tilde{x}[n]=\sum_{r=-\infty}^{\infty} x_{m}[n-\beta / 2-2 N r] w_{R X}[n-2 N r] .
$$

The $\beta / 2$ delay introduced by the windowing can be easily compensated by the frequency equalizer (FEQ).

The raised-cosine window given by

$$
w_{R X}[n]=\left\{\begin{array}{l}
\frac{1}{2}\left[1+\cos \left(\frac{\pi(n-\beta / 2)}{\beta+1}\right)\right] \quad-\beta / 2 \leq n \leq \beta / 2-1 \\
1 \quad \beta / 2 \leq n \leq 2 N-\beta / 2-1, \\
\frac{1}{2}\left[1+\cos \left(\frac{\pi\left(n-L_{R X}+1\right)}{\beta+1}\right)\right] \quad L_{R X} \leq n \leq L_{R X}+\beta-1
\end{array}\right.
$$

with $L_{R X}=2 N-\beta / 2$, has been employed in this work.

The out-band power of the transmitted signal can be also reduced by using pulse-shaping [4]. To this end, DMT symbols are periodically extended $\alpha$ samples at both ends, multiplied by the a pulse-shaping window, $w_{T X}[n]$, and overlapped in time. The resulting signal can then be expressed as

$$
x[n]=\sum_{m=-\infty}^{\infty} \sum_{k=-(N-1)}^{N} X_{m, k} e^{j \frac{\pi}{N} k\left(n-c p-\alpha-m L_{T X}\right)} w_{T X}\left[n-m L_{T X}\right],
$$

where $L_{T X}=2 N+c p+\alpha$.

Since the IDFT-based transmitter can still be used, the price to be paid is essentially the reduction in the symbol rate and in the available energy at the receiver detector.

In this work, the raised-cosine window given by (6) has been employed.

$$
w_{T X}[n]=\left\{\begin{array}{ll}
\frac{1}{2}\left[1+\cos \left(\frac{\pi(n-\alpha)}{\alpha+1}\right)\right] & 0 \leq n \leq \alpha-1 \\
\frac{1}{2}\left[1+\cos \left(\frac{\pi\left(n-L_{T X}+1\right)}{\alpha+1}\right)\right] & \alpha \leq n \leq L_{T X}-1 \\
L_{\mathrm{TX}} \leq n \leq L_{T X}+\alpha-1
\end{array} .\right.
$$

\section{Carrier allocation strategy}

The optimal carrier allocation strategy in a frequencyselective environment is the solution to a non-linear optimization problem which is usually approximated by means of linear or integer programming [13]. In the case of DMTFDMA the problem becomes even more complicated, since the signal-to-noise-and-distortion (SNDR) experienced by each carrier depends on the assignation of the remaining carriers.

This work analyzes the MAI resulting from a carrier allocation strategy in which carriers are assigned individually and on a group basis. In both cases the number of active links is fixed to 2 , and the performance degradation is measured in terms of the number of subbands. Concretely, the $0 \mathrm{~Hz}$ to $30 \mathrm{MHz}$ band has been divided in 16, 32 and 64 subbands.

Three methods have been employed to assign subbands or individual carriers to links. The latter assignment problem is solved by means of linear programming (LP). The optimality criterion is to maximize the aggregate bit-rate of the two links, subject to the restriction that each of them must achieve, at least, $40 \%$ of the bit rate that they would obtain in a singleuser scenario [14]. For the subband assignment problem two alternatives have been used. The first one employs integer programming (IP) with the same optimality criterion employed for the individual carrier assignment problem. The second one assigns adjacent subbands to different users (interleaved), which provides the lower bound for the achievable performance of these schemes. A flat power spectral density (PSD) restriction is assumed in all cases.

\section{DISTORTION EVALUATION}

The whole distortion suffered by each user in a DMTFDMA system can be essentially divided into two terms: ISI and ICI. MAI is a particular type of ICI in which the interferer and interfered carriers belong to different users. An exact analytical expression for both terms can be derived when the channel response can be modeled as an LTI system. A detailed analysis will be firstly derived for the pulse shaped and windowed DMT system. Afterwards, expressions for the conventional DMT system will be obtained as a particular case of the former.

\section{A. DMT system with pulse-shaping and windowing}

A DMT system with pulse-shaping at the transmitter and windowing at the receiver can be modeled by the diagram shown in Fig. 2. 


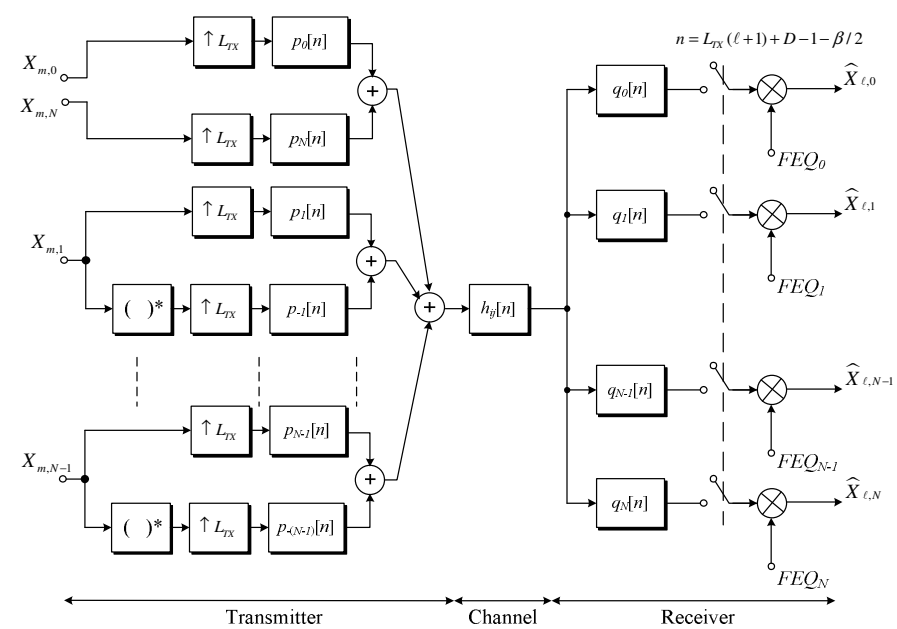

Fig. 2. Discrete-time model of a DMT system.

Symbols transmitted in carriers 0 and $N$ are real valued to force the transmitted signal to be a real sequence. $\widehat{X}_{\ell, z}$ is the $\ell$-th equalized output of carrier $z, F E Q_{z}$ is the one-tap frequency equalizer for carrier $z$ and ()$^{*}$ denotes the complex conjugate. The transmitter and receiver pulses are respectively given by

$$
p_{k}[n]=\frac{1}{2 N} e^{j \frac{\pi}{N} k(n-c p-\alpha)} w_{T X}[n],
$$

and

$$
q_{z}[n]=e^{j \frac{\pi}{N} z(n+1)} w_{R X}[n] .
$$

$h_{i j}[n]$ is the channel impulse response between the transmitter of user 1-link $i$ to the receiver of user 2-link $j$. $D$ is the starting sample of the effective impulse response, i.e. the $(c p+1)$-samples window of the impulse response with maximum energy [15].

The useful signal term in $\widehat{X}_{\ell, z}$ is obtained when $i=j$, $z=k$ and $\ell=m$. Fortunately, the effective length of indoor power-line channels is shorter than the symbol length of DMT systems that employs the band up to $30 \mathrm{MHz}$ with a reasonable number of carriers (e.g. $N>256$ ) [3]. This considerably simplifies the calculation of the distortion terms. Hence, the ISI is obtained when $i=j, z=k$ and $\ell=m-1, \ell=m+1$. The ICI when $i=j, \quad z \neq k$ and $\ell=m-1, \quad \ell=m$ and $\ell=m+1$. Finally, the MAI term is obtained when $i \neq j, z \neq k$ and $\ell=m-1, \ell=m$ and $\ell=m+1$.

Let's consider a one-shot transmission with index $m$ in the desired link. Denoting by

$$
b_{k}[n]=p_{k}[n] * h_{i i}[n]=b_{-k}[n]^{*},
$$

the output of carrier $z$ at $\ell=m-1$ can be expressed as

$$
\begin{aligned}
& \widehat{X}_{m-1, z}=2 \sum_{k=0}^{N}\left[X_{m, k}^{I}\left(\sum_{n=0}^{D-1} \operatorname{Re}\left[b_{k}[n]\right] w_{R X}\left[W_{-1}-n\right] e^{-j \frac{\pi}{N} z n}\right)\right. \\
& \left.+X_{m, k}^{Q}\left(\sum_{n=0}^{D-1}-\operatorname{Im}\left[b_{k}[n]\right] w_{R X}\left[W_{-1}-n\right] e^{-j \frac{\pi}{N} z n}\right)\right] e^{j \frac{\pi}{N} z(D-\beta)} F E Q_{z}^{\prime},
\end{aligned}
$$

where $W_{-1}=D-\beta / 2-1, F E Q_{z}^{\prime}=e^{j \frac{\pi}{N} z(\beta / 2)} F E Q_{z}$ and $X_{k, m}^{I}$ and $X_{k, m}^{Q}$ are the in-phase and quadrature components of $X_{k, m}$. The bracketed expressions in (10) can be efficiently performed by means of the $2 N$-point FFT of the signals $\operatorname{Re}\left[b_{k}[n]\right] w_{R X}\left[W_{-1}-n\right]$ and $-\operatorname{Im}\left[b_{k}[n]\right] w_{R X}\left[W_{-1}-n\right]$ with $0 \leq n \leq D-1$ and padded with zeros.

Similarly, the output of the sampler of carrier $z$ at $\ell=m$ can be expressed as

$$
\begin{aligned}
& \widehat{X}_{m, z}=2 \sum_{k=0}^{N}\left[X_{m, k}^{I}\left(\sum_{n=0}^{2 N+\beta-1} \operatorname{Re}\left[b_{k}\left[n+M_{0}\right]\right] w_{R X}\left[W_{0}-n\right] e^{-j \frac{\pi}{N} z n}\right)\right. \\
& \left.+X_{m, k}^{Q}\left(\sum_{n=0}^{2 N+\beta-1}-\operatorname{Im}\left[b_{k}\left[n+M_{0}\right]\right] w_{R X}\left[W_{0}-n\right] e^{-j \frac{\pi}{N} z n}\right)\right] F E Q_{z}^{\prime},
\end{aligned}
$$

where $M_{0}=\alpha+c p+D-\beta, W_{0}=2 N+\beta / 2-1$. The bracketed expressions in (11) can now be efficiently computed by decimating by 2 the output of the $4 N$-point FFT of the signals $\operatorname{Re}\left[b_{k}\left[n+M_{0}\right]\right] w_{R X}\left[W_{0}-n\right]$ and $-\operatorname{Im}\left[b_{k}\left[n+M_{0}\right]\right] w_{R X}\left[W_{0}-n\right]$ with $0 \leq n \leq 2 N+\beta-1$ and padded with zeros.

Finally, the output of the sampler at $\ell=m+1$ are given by

$$
\begin{aligned}
& \widehat{X}_{m+1, z}=2 \sum_{k=0}^{N}\left[X_{m, k}^{I}\left(\sum_{n=0}^{L h-M_{0}+\alpha-2} \operatorname{Re}\left[b_{k}\left[n+M_{1}\right]\right] w_{R X}\left[W_{0}-n\right] e^{-j \frac{\pi}{N} z n}\right)\right. \\
& \left.+X_{m, k}^{Q}\left(\sum_{n=0}^{L h-M_{0}+\alpha-2}-\operatorname{Im}\left[b_{k}\left[n+M_{1}\right]\right] w_{R X}\left[W_{0}-n\right] e^{-j \frac{\pi}{N} z n}\right)\right] F E Q_{z}^{\prime},
\end{aligned}
$$

where $M_{1}=L_{T X}+M_{0}$. Correspondingly, the bracketed expressions in (12) can be also obtained by means of the $2 \mathrm{~N}$ point FFT of the signals $\operatorname{Re}\left[b_{k}\left[n+M_{1}\right]\right] w_{R X}\left[W_{0}-n\right]$ and $-\operatorname{Im}\left[b_{k}\left[n+M_{1}\right]\right] w_{R X}\left[W_{0}-n\right]$ with $0 \leq n \leq L_{h}-M_{0}+\alpha-2$ and padded with zeros.

Based on the above results, the input-output relation for the DMT system can be matricially written as

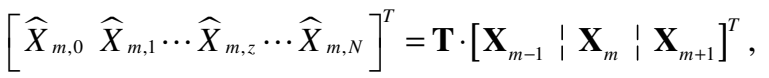

where $\widehat{X}_{m, z}$ denotes the $m$-th complex value received at the output of carrier $z, \mathbf{X}_{m-i}$ is the vector with the $(m+i)$-th transmitted values in each carrier

$$
\mathbf{X}_{m+i}=\left[X_{m+i, 0}^{I} X_{m+i, 0}^{Q} X_{m+i, 1}^{I} X_{m+i, 1}^{Q} \cdots X_{m+i, N-1}^{I} X_{m+i, N-1}^{Q} X_{m+i, N}^{I} X_{m+i, N}^{Q}\right],
$$

with $i=-1,0,1$ and where $\mathbf{T}$ is a transmission matrix defined as

$$
\mathbf{T}=\left[\begin{array}{l:l:l}
\mathbf{T}_{m-1} & \mathbf{T}_{m} & \mathbf{T}_{m+1}
\end{array}\right],
$$

with

$$
\mathbf{T}_{m+i}=\left[\mathbf{T}_{m+i, 0}^{I} \mathbf{T}_{m+i, 0}^{Q} \mathbf{T}_{m+i, 1}^{I} \mathbf{T}_{m+i, 1}^{Q} \cdots \mathbf{T}_{m+i, N-1}^{I} \mathbf{T}_{m+i, N-1}^{Q} \mathbf{T}_{m+i, N}^{I} \mathbf{T}_{m+i, N}^{Q}\right] .
$$

The elements of $\mathbf{T}_{m+i}$ are the column vectors

$$
\mathbf{T}_{m+i, k}^{I}=\left[\begin{array}{llllll}
T_{m+i, k}^{0, I} & T_{m+i, k}^{1, I} \cdots T_{m+i, k}^{z, I} & T_{m+i, k}^{z, I} \cdots T_{m+i, k}^{N-1, I} & T_{m+i, k}^{N, I}
\end{array}\right]^{T},
$$




$$
\mathbf{T}_{m+i, k}^{Q}=\left[\begin{array}{lllll}
T_{m+i, k}^{0, Q} & T_{m+i, k}^{1, Q} \cdots T_{m+i, k}^{z, Q} & T_{m+i, k}^{z, Q} \cdots T_{m+i, k}^{N-, Q} & T_{m+i, k}^{N, Q}
\end{array}\right]^{T},
$$

whose elements are the complex coefficient by which the inphase and quadrature values transmitted during the $(m+i)$-th symbol in carrier $k$ appear at the $m$-th output of carrier $z$. They can be obtained from the signals

$$
f_{k}^{M, W, P}=\left\{\begin{array}{cc}
\operatorname{Re}\left[b_{k}[n+M]\right] w_{R X}[W-n] & 0 \leq n \leq P \\
0 & \text { otherwise }
\end{array},\right.
$$

and

$$
g_{k}^{M, W, P}=\left\{\begin{array}{cl}
-\operatorname{Im}\left[b_{k}[n+M]\right] w_{R X}[W-n] & 0 \leq n \leq P \\
0 & \text { otherwise }
\end{array},\right.
$$

according to

$$
\begin{gathered}
\mathbf{T}_{m-1, k}^{z, I}=2 e^{j \frac{\pi}{N} z(D-\beta)} F E Q_{z}^{\prime} F F T\left(f_{k}^{0, W_{0}, D-1}, 2 N, z\right), \\
\mathbf{T}_{m-1, k}^{z, Q}=2 e^{j \frac{\pi}{N} z(D-\beta)} F E Q_{z}^{\prime} F F T\left(g_{k}^{0, W_{0}, D-1}, 2 N, z\right), \\
\mathbf{T}_{m, k}^{z, I}=2 F E Q_{z}^{\prime} F F T\left(f_{k}^{M_{0}, W_{0}, 2 N+\beta-1}, 4 N, 2 z\right), \\
\mathbf{T}_{m, k}^{z, Q}=2 F E Q_{z}^{\prime} F F T\left(g_{k}^{M_{0}, W_{0}, 2 N+\beta-1}, 4 N, 2 z\right), \\
\mathbf{T}_{m+1, k}^{z, I}=2 F E Q_{z}^{\prime} F F T\left(f_{k}^{M_{1}, W_{0}, L_{h}-M_{0}+\alpha-2}, 2 N, z\right), \\
\mathbf{T}_{m+1, k}^{z, Q}=2 F E Q_{z}^{\prime} F F T\left(g_{k}^{M_{1}, W_{0}, L_{h}-M_{0}+\alpha-2}, 2 N, z\right),
\end{gathered}
$$

with $0 \leq z \leq N$ and where $F F T\left(x[n], N^{\prime}, z\right)$ denotes

$$
F F T\left(x[n], N^{\prime}, z\right)=\sum_{n=0}^{N^{\prime}-1} x[n] e^{-j \frac{2 \pi}{N^{\prime}} n z}, \quad 0 \leq z \leq N^{\prime} .
$$

\section{B. Conventional DMT system}

By particularizing $\alpha=\beta=0$ in Fig. 2 and following an analogous procedure, the coefficients of the transmission matrix for the conventional DMT system can be obtained from

$$
\begin{gathered}
\mathbf{T}_{m-1, k}^{z, I}=2 e^{j \frac{\pi}{N} z D} F E Q_{z} F F T\left(f_{k}^{0, D-1}, 2 N, z\right), \\
\mathbf{T}_{m, k}^{z, I}=2 F E Q_{z} F F T\left(f_{k}^{M_{0}, 2 N-1}, 2 N, z\right), \\
\mathbf{T}_{m+1, k}^{z, I}=2 F E Q_{z} F F T\left(f_{k}^{M_{1}, L_{h}-c p-D-2}, 2 N, z\right),
\end{gathered}
$$

with $0 \leq z \leq N$ and

$$
f_{k}^{M, P}=\left\{\begin{array}{cl}
\operatorname{Re}\left[b_{k}[n+M]\right] & 0 \leq n \leq P \\
0 & \text { otherwise }
\end{array} .\right.
$$

The corresponding values of $\mathbf{T}_{m+i, k}^{z, Q}$ can be obtained by computing (27), (28) and (29) over the signal

$$
g_{k}^{M, P}=\left\{\begin{array}{cl}
-\operatorname{Im}\left[b_{k}[n+M]\right] & 0 \leq n \leq P \\
0 & \text { otherwise }
\end{array},\right.
$$

\section{Signal-to-distortion ratio (SDR) calculation}

The transmission matrix $\mathbf{T}$ allows exact computation of the different distortion terms. Assuming that the in-phase and quadrature components of the complex values transmitted in carrier $z$ are independent with power $\sigma_{z, I}^{2}$ and $\sigma_{z, Q}^{2}$, the ISI in term in carrier $z$ is given by

$$
I S I_{z}=\left[\left(\left|T_{z, m-1}^{z, I}\right|^{2}+\left|T_{z, m+1}^{z, I}\right|^{2}\right) \sigma_{z, I}^{2}+\left(\left|T_{z, m-1}^{z, Q}\right|^{2}+\left|T_{z, m+1}^{z, Q}\right|^{2}\right) \sigma_{z, Q}^{2}\right],
$$

Similarly, the ICI can be obtained from

$$
\begin{aligned}
& I C I_{z}=\sum_{\substack{k=0 \\
k \neq z}}^{N}\left[\left(\left|T_{k, m-1}^{z, I}\right|^{2}+\left|T_{k, m}^{z, I}\right|^{2}+\left|T_{k, m+1}^{z, I}\right|^{2}\right) \sigma_{k, I}^{2}\right. \\
& \left.+\left(\left|T_{k, m-1}^{z, Q}\right|^{2}+\left|T_{k, m}^{z, Q}\right|^{2}+\left|T_{k, m+1}^{z, Q}\right|^{2}\right) \sigma_{k, Q}^{2}\right],
\end{aligned}
$$

which also provides the MAI when the transmission matrix is computed employing the interferer channel, $h_{i j}[n]$.

The SDR in carrier $z$, which is usually defined as

$$
S D R_{z}=\frac{E\left[\left|X_{z, m}\right|^{2}\right]}{E\left[\left|\widehat{X}_{z, m}-X_{z, m}\right|^{2}\right]},
$$

can then be expressed in terms of the ISI and ICI as

$$
S D R_{z}=\frac{\sigma_{z, I}^{2}+\sigma_{z, Q}^{2}}{I S I_{z}+I C I_{z}+\left(\left|1-T_{m, z}^{z, I}\right|^{2} \sigma_{z, I}^{2}+\left|j-T_{m, z}^{z, Q}\right|^{2} \sigma_{z, Q}^{2}\right)} .
$$

When the cyclic prefix length is longer than the channel impulse response, $T_{m, z}^{z, I}=1$ and $T_{m, z}^{z, Q}=j$, and the bracketed terms in the denominator of (36) are zero. However, when an insufficient cyclic prefix length is used, the channel effect over the received symbols can not be modeled as a multiplication by a complex value, not even in a one shot transmission. Hence, these terms reflect the impossibility of performing a perfect equalization with a one-tap FEQ.

The presented method also allows to assess the SDR caused by a frequency mismatch of $\Delta f(\mathrm{ppm})$ in a symbol synchronized network just by modifying the transmitted pulse

$$
p_{k}[n]=\frac{1}{2 N} e^{j \frac{\pi}{N} k(n(1+\Delta f)-c p-\alpha)} w_{T X}[n(1+\Delta f)] .
$$

The analytical procedure presented in this section is a powerful tool for the study of carrier allocation strategies, since the ICI caused by a single carrier can be easily computed. This approach becomes particularly useful in multiuser environments where, once the transmission matrices have been computed, the SDR for different carrier assignments can be obtained with a reduced computational load employing expressions (36). The implementation complexity of the presented method for the conventional DMT system is essentially that of the $N$ convolutions and the $6 N$ FFT's of $2 N$ points (slightly higher for the DMT system with pulse shaping and windowing). In a conventional system simulation, the transmission of $M$ symbols requires $M$ convolutions and $2 M$ FFT's of $2 N$ points.

\section{PERFORMANCE RESULTS}

\section{A. Indoor power-line scenario}

In order to asses the performance of the DMT-FDMA scheme, three transmitters and three receivers have been distributed in an apartment of about $80 \mathrm{~m}^{2}$. Receivers have been numbered 1, 3 and 5, while 2, 4 and 6 have been used for 
transmitters. Numbers have been assigned according to the estimated physical length of the main path between transmitter and receiver, e.g. the main path between receiver 1 and transmitter 2 is much shorter than the one between receiver 1 and transmitter 6 .

Three MAI scenarios, each one with two unidirectional links have been defined. Table I shows the transmitters and receivers positions of each one.

TABLE I

MAI SCENARIOS

\begin{tabular}{cc}
\hline \hline $\begin{array}{c}\text { Desired Link } \\
\text { Transmitter/Receiver position }\end{array}$ & MAI Category Scenario \\
\hline $6 / 5$ & Best \\
$2 / 1$ & Intermediate \\
\hline $4 / 5$ & Worst \\
$6 / 3$ & \\
$2 / 1$ & \\
\hline \hline
\end{tabular}

Fig. 3 shows the frequency responses of the desired and interferer channels and Fig. 4 the PSD at the receivers' sites.

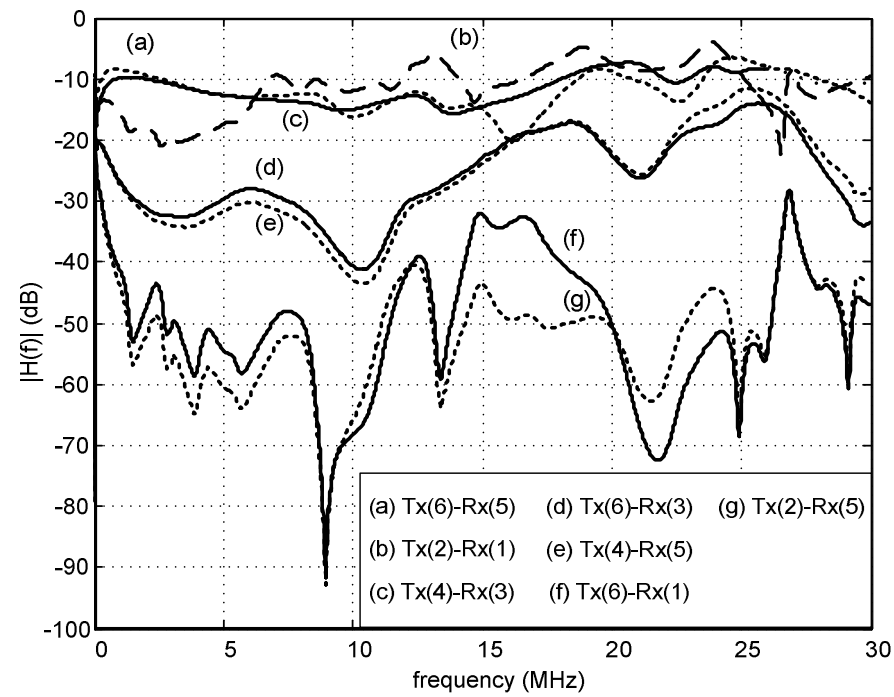

Fig. 3. Frequency response of the desired and interferer channels.

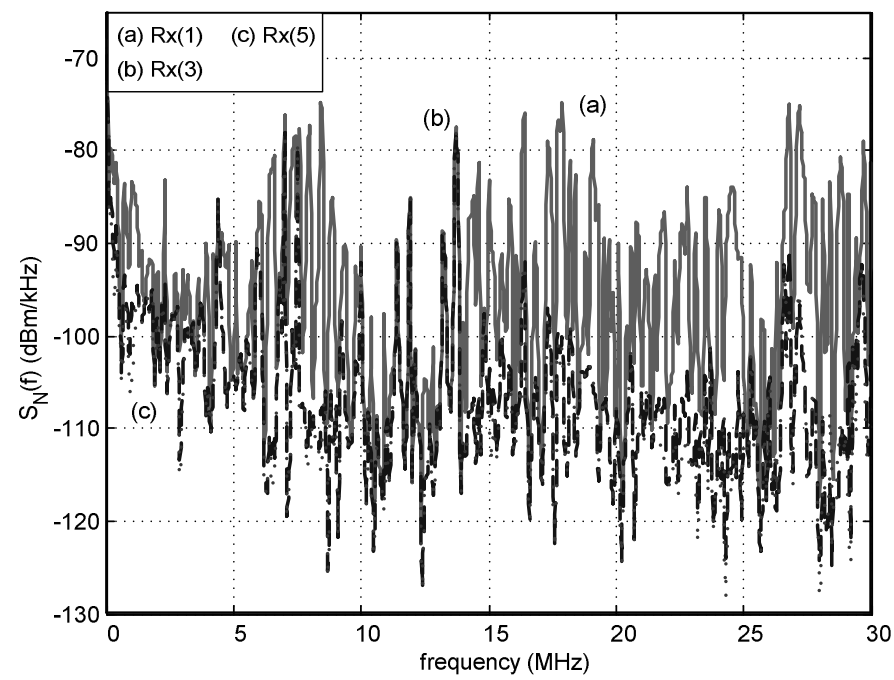

Fig. 4. Noise PSD at the three receivers' site.
Fig. 3 shows that the best scenario presents lowly attenuated desired channels and highly attenuated interferer channels. The reverse occurs in the worst scenario. Regarding the noise PSD, except for some minor differences around $1 \mathrm{MHz}$, the values for receivers 3 and 5, are practically equal.

\section{B. Parameterization of the DMT systems}

Before comparing the performance of the conventional DMT system with that of the DMT system with pulse-shaping and windowing, they must be properly parameterized. Table II shows the parameters employed in the conventional DMT system. The number of carriers and the cyclic prefix length have selected according to the results presented in [2]. No coding is used. The DMT system with pulse-shaping and windowing also employs the parameters in Table II, except for the cyclic prefix length, which is $\beta$ samples longer.

Optimum values of $\alpha$ and $\beta$ are strongly dependent on the importance of the MAI. Hence, the selection of the most appropriate values for both of them is carried out separately for the cases of symbol misalignment and frequency asynchrony.

\section{TABLE II}

DMT SYSTEM PARAMETERS

\begin{tabular}{cc}
\hline \hline Parameter & Value \\
\hline Sampling frequency $\left(f_{s}\right)$ & $60 \mathrm{MHz}$ \\
Number of carriers $(N)$ & 1024 \\
Ciclix prefix length $(c p)$ & $228 \mathrm{samples}$ \\
Transmission PSD & $-20 \mathrm{dBm} / \mathrm{kHz}$ \\
Constellations & Square QAM and BPSK \\
Maximum number of bits per QAM & 16 \\
symbol & $10^{-6}$ \\
Symbol error rate (Pe) & \\
\hline \hline
\end{tabular}

\section{Symbol misalignment}

In the case of an integer symbol displacement, increasing $\alpha$ and $\beta$ reduces the ICI and the MAI at the receiver but the symbol rate and the available energy for the detection process is also reduced. MAI is minimum in the case of symbol synchronization, where it is exclusively due to channel dispersion, and maximum when the misalignment between the desired and interferer symbols is about one half of the symbol length. Therefore, the selection of $\alpha$ and $\beta$ is performed in the latter situation. To this end, the bit-rate of the six links has been evaluated for the different carrier assignment. Since the ICI and the MAI are caused by the contribution of a high number of independent sources, they have been assumed to be gaussian.

In scenarios in which the pulse-shaping and the windowing improve the performance, it has been observed that the bit-rate is maximized when $\alpha \approx \beta$. As an example, Fig. 5 shows the bit-rate values obtained for one link of the intermediate MAI scenario (4/5) for a carrier assignment consisting of 16 interleaved subbands. It can be also observed that for small values of $\alpha$ and $\beta$, small increments in these parameters result in considerable bit-rate improvements. On the other hand, 
when the reduction in the MAI power, which allows to use denser constellations, does not compensate for the reduction in the symbol rate, the bit-rate decreases when $\alpha$ or $\beta$ are increased.

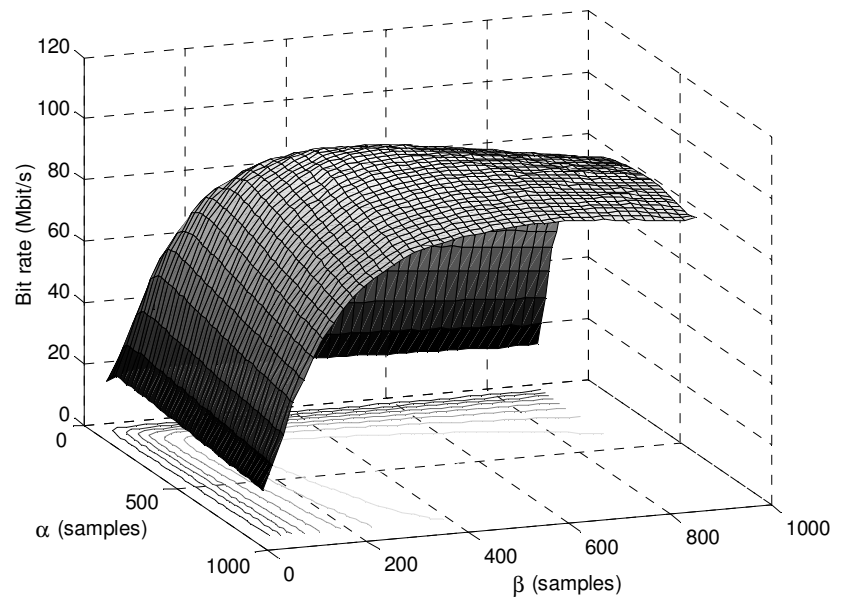

Fig. 5. Bit-rate for a link of the intermediate MAI scenario with 16 interleaved subbands.

To select the most appropriate values for $\alpha$ and $\beta$, the interleaved subbands carrier assignments schemes have been selected, since they provide upper bounds for the MAI. Fig. 6 depicts the aggregate bit-rate of each MAI scenario for the 16 and 64 interleaved subbands schemes when $\alpha=\beta$ and a worst symbol misalignment situation.

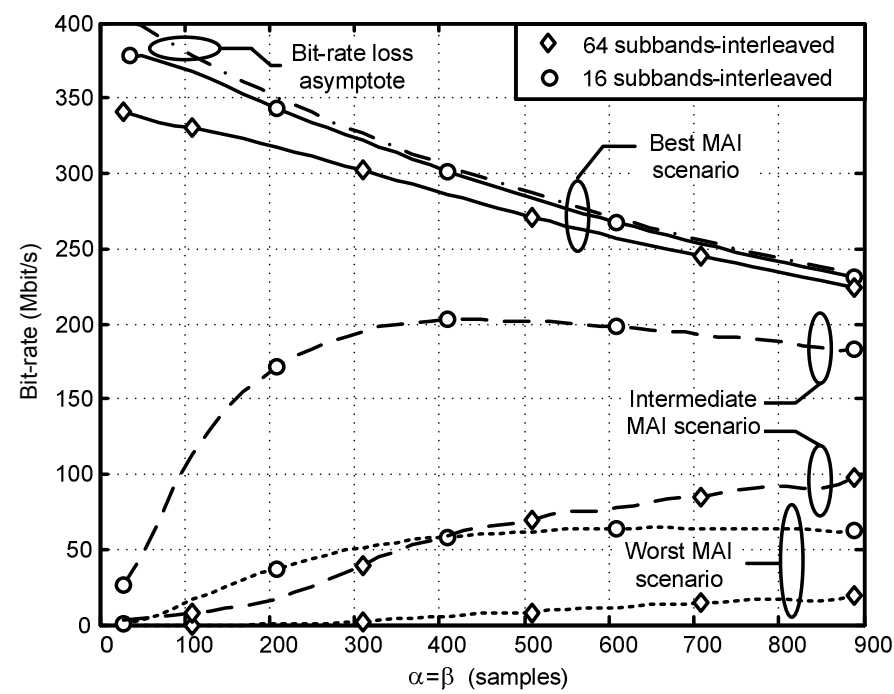

Fig. 6. Aggregate bit-rate of each MAI scenario as a function of $\boldsymbol{\alpha}$ and $\boldsymbol{\beta}$.

As seen, in the best MAI scenario pulse-shaping and windowing is nearly always counterproductive, since the MAI is very small. This fact is reinforced by noting that the bit-rate for the 16 interleaved subbands scheme is nearly coincident with the bit-rate asymptote due to symbol rate loss. This asymptote has been calculated by summing the bit-rates obtained in this scenario when only one user is active at a time and scaling the symbol rate with the corresponding values of $\alpha$ and $\beta$. This result also reveals the comparatively reduced influence of the energy loss in the bit-rate.

It can be also observed that the bit-rate loss in the intermediate and worst MAI scenarios has a very similar behavior. In the 16 interleaved subbands scheme there is an optimum value for $\alpha$ and $\beta$ around 500 samples. On the contrary, in the 64 interleaved subbands case, the MAI is so strong that increasing $\alpha$ and $\beta$ in the analyzed range always improves the performance.

Fig. 6 shows that $\alpha=\beta=500$ samples would be an appropriate trade-off for most situations. Consequently, these have been the selected values for the pulse-shaping and windowing. The performance of the system parameterized in this way is now compared with that of the conventional DMT system. To this end, the aggregate bit-rate achieved with these systems in each scenario has been obtained as a function of the symbol misalignment, $\Delta n$. Fig. 7 depicts the bit-rate loss for the worst MAI scenario. The sum of the individual bit-rates obtained by the conventional DMT system in the same scenarios with only one link active at a time is taken as reference.

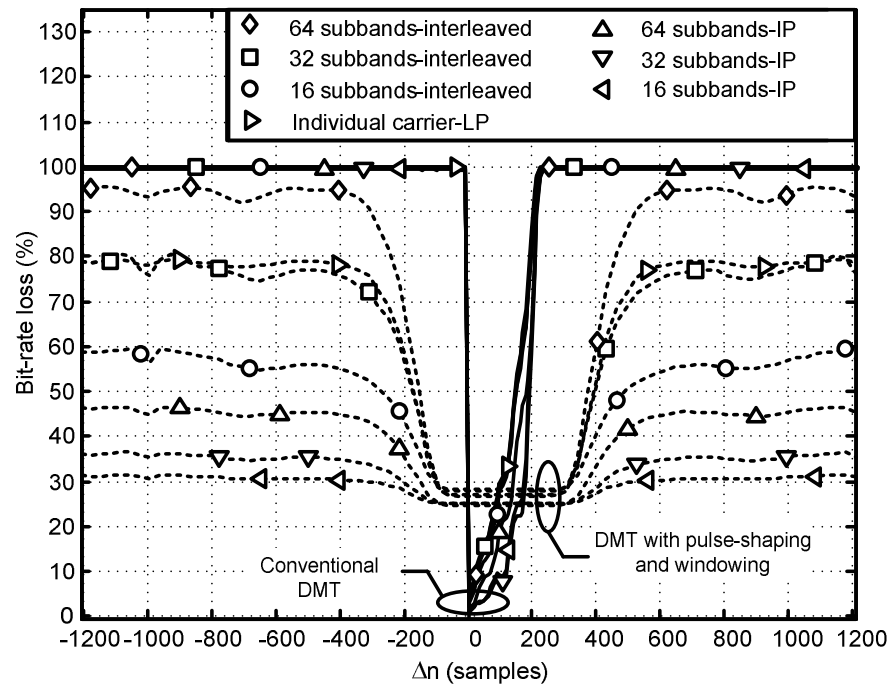

Fig. 7. Bit-rate loss for the worst MAI scenario as a function of the symbol misalignment.

It is interesting to note that the effect of a time advance $(\Delta n<0)$ in the interferer symbol is much harmful than a delay of the same magnitude. The reason is that in the latter situation, the interferer's symbol transitions occurs within the $\mathrm{cp}$ of the desired symbol, which absorbs part of the generated MAI. It can be also observed that the performance of the conventional DMT system is extremely sensitive to small symbol misalignments. This suggests that in order to relax the requisites of an FDMA network synchronization system, the displacement of the symbol synchronizers with respect to their optimum position in a single-user environment may be an interesting option.

Fig. 7 also reveals that performance degradation due to channel dispersion is negligible in a synchronized conventional DMT system. Regarding the DMT system with pulse-shaping and windowing, it is shown that the bit-rate loss is around $25 \%$ in a symbol synchronized situation. However, in the presence of symbol misalignment, the bit-rate becomes 
much more resilient to the MAI. It is also important to note the impact of the carrier assignment in the performance degradation. Thus, whereas in the case of 16 subbands assigned with IP the loss is around 30\%, in the case of 64 interleaved subbands the communication system is nearly on outage.

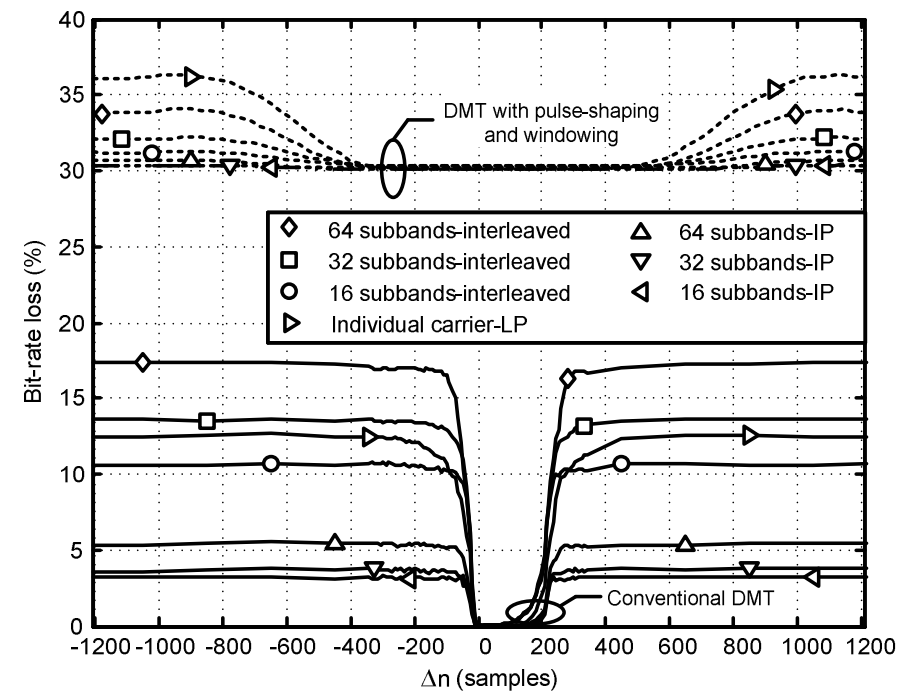

Fig. 8. Bit-rate loss for the best MAI scenario as a function of the symbol misalignment.

The corresponding values for the bit-rate loss in the best MAI scenario are shown in Fig. 8. This time, the MAI is so small that pulse-shaping and windowing are always counterproductive. However, even with a $30 \%$ loss, the aggregate bit-rate achieved in this scenario is over $250 \mathrm{Mbit} / \mathrm{s}$.

\section{Frequency asynchrony}

In this section, symbol misalignment is assumed to be kept less than one sample, what is feasible in a symbol synchronized network in which periodical synchronizations beacons are sent at a proper rate. In this scenario, terminals would adjust the phase of their clocks after the reception of the beacon. However, in the interval between two consecutive synchronization beacons, users' frequency drift cause MAI. In these circumstances, pulse-shaping at the transmitter is nearly always counterproductive, since it only reduces the MAI caused by channel dispersion. Hence, only windowing is considered.

In order to determine the most appropriate value for $\beta$, the aggregate bit-rate of the three scenarios have been estimated for a worst case frequency mismatch of $\Delta f=25 \mathrm{ppm}$. Fig. 9 depicts the bit-rate values for the 16 and 64 interleaved subbands schemes versus $\beta$. As observed, the behavior is quite similar to the one seen in the case of symbol asynchrony. In the best MAI scenario windowing is always counterproductive and in the rest of the cases it improves the bit-rate in all situations. A trade-off value of $\beta=480$ has been selected for successive analyses.

Fig. 10 depicts the bit-rate loss for the best and worst MAI scenario as a function of the frequency mismatch. The sum of the individual bit-rates obtained by the conventional DMT system in the same scenarios with only one link active at a time is taken as reference.

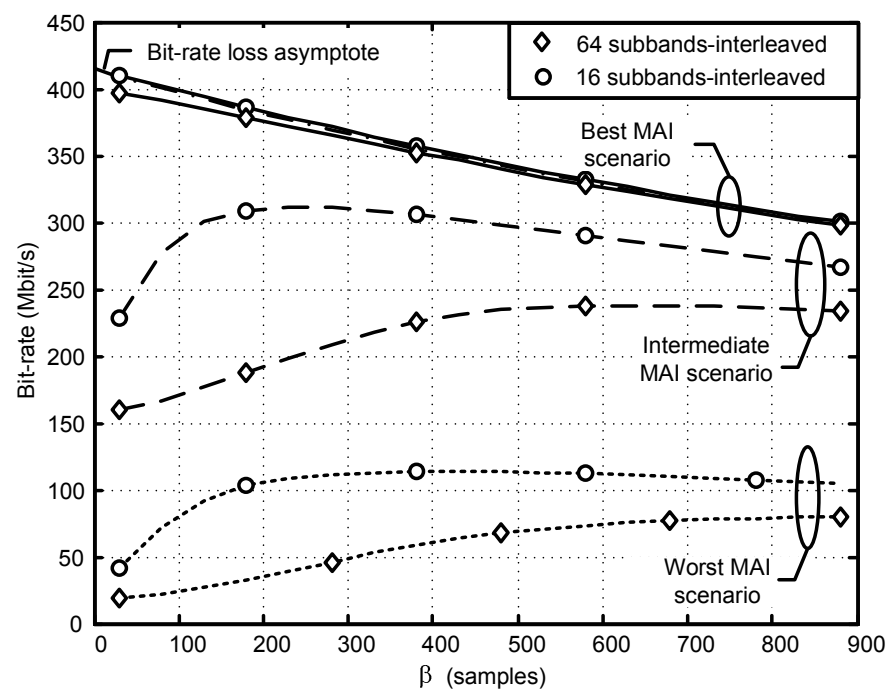

Fig. 9. Aggregate bit-rate of each MAI scenario as a function of $\beta$.

It can be observed that in the best MAI scenario the performance of the conventional DMT system is nearly unaffected by the frequency mismatch. On the other hand, in the worst MAI scenario, its performance is seriously degraded even for small frequency offsets. In this scenario, the bit-rate loss of the windowed DMT may also be considerable for some carrier assignment even for a frequency mismatch of $30 \mathrm{ppm}$. However, for frequency errors smaller than $2 \mathrm{ppm}$, the bit-rate loss is below $30 \%$, which ensures more than $110 \mathrm{Mbit} / \mathrm{s}$. In the best MAI scenario, although not shown in the figure, the bitrate loss of the windowed DMT system in the analyzed range is around $15 \%$.

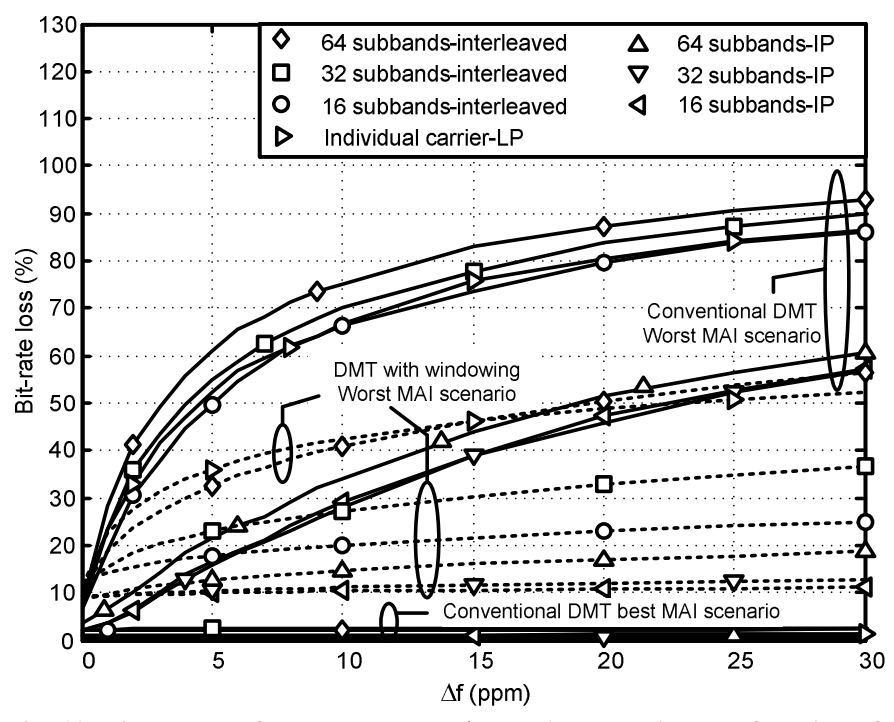

Fig. 10. Bit-rate loss for the worst and best MAI scenarios as a function of the frequency mismatch.

\section{CONCLUSIONS}

This paper has presented a novel analytical procedure to estimate the different distortion terms (ISI, ICI and MAI) of a DMT system operating over an LTI channel. This method has 
been employed to analyze a DMT-FDMA scheme for broadband indoor power-line communications. Performance degradation due to the MAI caused by the frequency selectivity of the channel, symbol misalignment and frequency mismatch has been evaluated.

It has been shown that the bit-rate loss due to the MAI is negligible in a symbol and frequency synchronized scheme. However, its performance is extremely sensitive to small asynchronies. System outage may occur even for less than ten samples of symbol misalignment or frequency mismatches of 40ppm.

The use of pulse-shaping at the DMT transmitter and windowing at the receiver, in conjunction with a carrier allocation scheme in which tones are assigned to users on a group basis, has been studied as a mean for implementing an asynchronous FDMA scheme. The effect of symbol misalignment is so harmful that, even in these circumstances, bit-rate losses can be up to $60 \%$ in a network with 16 subbands and up to $80 \%$ in the case of 32 subbands. In the presence of frequency errors pulse-shaping is counterproductive in nearly all situations and, although windowing has proven to be an effective way of reducing performance degradation, bit-rate losses of up to $35 \%$ can occur for $20 \mathrm{ppm}$ of frequency error.

\section{REFERENCES}

[1] H. Philipps, "Performance measurements of power-line channels at high frequencies," in Proc. of the International Symposium on Power-Line Communications and its Applications (ISPLCA), 1998, pp. 229-237.

[2] F. J. Cañete, J. A. Cortés, L. Díez, and J. T. Entrambasaguas, "Modeling and evaluation of the indoor power line channel," IEEE Communication Magazine, Vol. 41, pp. 41-47, April 2003.

[3] F. J. Cañete, J. A. Cortés, L. Díez and J. T. Entrambasaguas, "Analysis of the Cyclic Short-Term Variation of Indoor Power-line Channels," IEEE Journal on Selected Areas on Communications, Vol. 24, Issue 7, pp. 1327-1338, July 2006.

[4] O. Edfors et al., "An Introduction to Orthogonal-frequency Division Multiplexing,” Res. rep. 1996:16, Div.of Sig. Processing, Luleå Univ. of Tech., Sept. 1996.

[5] The Homeplug Powerline Alliance, "Homeplug AV White Paper," http://www.homeplug.org

[6] The Open PLC European Research Alliance (Opera), http://www.istopera.org

[7] P. G. Flikkema, "On multiple access and capacity in frequency-selective channels," in Proc. of the IEEE International Conference on Information Technology, Coding and Computing, 2001 pp. 178-182.

[8] J. J. van de Beek et al., "A time and frequency synchronization scheme for multiuser OFDM," IEEE Journal on Selected Areas in Communications, Vol. 17, No. 11, Nov. 1999, pp. 1900-1914.

[9] F. Sjöberg, R. Nilsson, M. Isaksson, P. Ödling, P. O. Börjesson, "Asynchronous Zipper," Proceedings of the IEEE International Conference on Communications, June 1999, Vancouver (Canada), pp. 231-235.

[10] J. Cioffi et al, "ADC precision contributions"- See BOTH ANSI T1E1.4 contributions 99-274R2 (June, 1999, Ottawa) and 99-274R3 (August, 1999 Baltimore).

[11] T. Banwell, S. Galli, "On the symmetry of the power line channel," International Symposium on Power Line Communications and Its Applications, ISPLC 2001, Malmo (Sweden), April 4-6, 2001.

[12] P. Spruyt, P. Reusens, S. Braet, "Performance of improved DMT transceiver for VDSL," Tech. Rep. T1E1.4/96-104, ANSI, Colorado Springs, CO, Apr. 1996.

[13] W. Yu, J. M. Cioffi, "FDMA Capacity of gaussian multiple-access channels with ISI," IEEE Transactions on Communications, Vol. 50, No. 1, pp. 102-111, Jan. 2002.
[14] T. Sartenaer, L. Vandendorpe, J. Louveaux, "Balanced capacity of wireline multiuser channels," IEEE Transactions on Communications, Vol. 53, Issue 12, pp. 2029-2042, Dec. 2005.

[15] T. Pollet and M. Peeters, "Synchronization with DMT modulation," IEEE Communications Magazine, Vol. 37, issue 4, pp 80-86, April 1999.

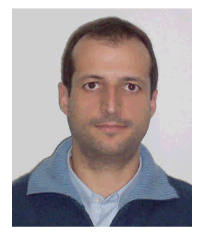

José Antonio Cortés Arrabal received the M.S. degree in telecommunication engineering from the University of Málaga (Spain) in 1998. In 1998 he received a fellowship from the R\&D department of Alcatel Citesa (Málaga). In 1999 he joined the Communication Engineering Department of the University of Málaga, where he is currently working toward the Ph.D. degree. From 2000 to 2002 he collaborated with the Nokia System Competence Team in Málaga. His research interests include digital signal processing for communications, mainly focused on synchronization and transmission techniques for high-speed power line communications.

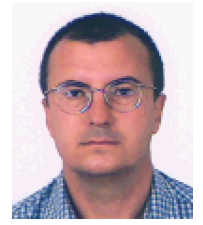

Luis Díez del Río received the M.S. and Ph.D. degrees from the Polytechnic University of Madrid, Spain, in 1989 and 1995, respectively, both in telecommunications engineering. In 1984, he joined Fujitsu-España R\&D center, where he worked in the development of modems. From 1987 to 1997 , he was with the Department of Signals, Systems and Radiocommunication, Polytechnic University of Madrid. Since 1997, he has been with the Communication Engineering Department, University of Málaga. His research interests include digital communication, a field in which he has worked for many years. His experience includes most of its applications, i.e., voice band, DSL and cable modems, satellite, mobile, and power line communications, etc., as well as technical aspects, i.e., synchronization, adaptive signal processing, modulation, coding and multiple access.

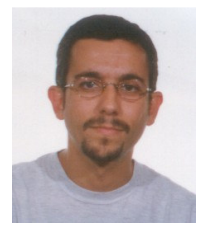

Francisco Javier Cañete Corripio received the M.S. and Ph.D. degrees in telecommunication engineering in 1996 and 2004, respectively, from the University of Málaga (Spain). In 1996, he worked for the Instrument and Control department at the Empresa Nacional de Ingeniería y Tecnología (INITEC) in the design of power plants. In 1997, He worked for Alcatel España R\&D department in the design of wireless local loop systems. Since 1998 he works for the Communication Engineering Department, at University of Málaga. From 2000 to 2001 he also collaborated with the Nokia System Competence Team in Málaga. His research activity is focused on digital signal processing for digital communications and his interests include channel modelling and transmission techniques for wireless systems and power- line communication systems.

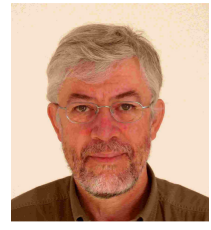

José Tomás Entrambasaguas Muñoz received the M.S. and Ph.D. degrees, in 1975 and 1990, respectively, both from the Polytechnic University of Madrid, Spain. From 1975 to 1978, he worked at the Polytechnic University of Madrid. In 1978 he joined Fujitsu-España R\&D center, where he worked in the development of packet switching systems for data networks (in collaboration with Telefónica de España), data transmission systems, and computer local area networks. In 1993, he joined the University of Málaga at the Communication Engineering Department, where his current interests include digital signal processing techniques applied to digital communication and methodologies for efficient and integrated development of complex communications systems. 